\title{
Right Ventricular Shape Distortion in Tricuspid Regurgitation
}

\author{
Ashley E Morgan ${ }^{1}$, Atefeh Kashani ${ }^{1}$, Brian Zenger ${ }^{1}$, Lindsay C Rupp ${ }^{1}$, Maura D Perez ${ }^{1}$, Markus D \\ Foote $^{1}$, Alan K Morris ${ }^{1}$, Mark B Ratcliffe ${ }^{2}$, Jiwon J Kim ${ }^{3}$, Jonathan W Weinsaft ${ }^{3}$ Vikas Sharma $^{1}$, \\ Rob S MacLeod ${ }^{1}$, Shireen Elhabian ${ }^{1}$ \\ ${ }^{1}$ University of Utah, Salt Lake City, UT, USA \\ ${ }^{2}$ University of California, San Francisco, and the San Francisco VA Medical Center \\ ${ }^{3}$ Weill-Cornell Medical College, NYC, NY, USA
}

\begin{abstract}
Tricuspid regurgitation (TR) is a failure in right-sided $A V$ valve function which, if left untreated, leads to marked cardiac shape changes and heart failure. However, the specific right ventricular shape changes resulting from $T R$ are unknown. The goal of this study is to characterize the RV shape changes of patients with severe TR. RVs were segmented from CINE MRI images. Using particlebased shape modeling (PSM), a dense set of homologous landmarks were placed with geometric consistency on the endocardial surface of each $R V$, via an entropybased optimization of the information content of the shape model. Principal component analysis (PCA) identified the significant modes of shape variation across the population. These modes were used to create a patient-prediction model. 32 patients and 6 healthy controls were studied. The mean RV shape of TR patients demonstrated increased sphericity relative to controls, with the three most dominant modes of variation showing significant widening of the short axis of the heart, narrowing of the base at the $R V$ outflow tract (RVOT), and blunting of the RV apex. By $P C A$, shape changes based on the first three modes of variation correctly identified patient vs. control hearts $86.5 \%$ of the time. The shape variation may further illuminate the mechanics of TR-induced $R V$ failure and recovery, providing potential targets for therapies including novel devices and surgical interventions.
\end{abstract}

\section{Introduction}

Tricuspid regurgitation (TR) occurs when the leaflets of the tricuspid valve separating the right atrium and right ventricle (RV) do not seal completely, allowing back-flow of blood from the RV to the right atrium during systole. TR is caused by a diverse set of disorders - including congenital and acquired anomalies of the tricuspid valve, pulmonary vascular disease, and left heart failure. Once TR arises, it triggers an ongoing cycle of right-sided volume overload, RV dilation, and worsening TR. This cycle leads to progressive RV dysfunction and remodeling, and ultimately RV failure. Approximately 1.6 million Americans currently live with moderate or severe TR, a number increasing consistently over time $[1,2]$. Severe TR is associated with significant risks, including an increased incidence of heart failure, 3-4-fold increased risk of major adverse cardiac events, and 2-3-fold risk of death [3-5].

Current research has shown that RV shape is correlated with function, and shape changes are associated with known markers of cardiovascular disease. For example, studies of pulmonary hypertension have shown increased RV eccentricity, while increased RV sphericity is associated with systemic hypertension, obesity, and smoking. Other studies have shown that shape changes correlate with symptom severity in patients with the congenital malformation of a dominant single ventricle [6-8]. However, the RV shape changes resulting from significant TR have not been previously described. Furthermore, these existing studies use crude approximations of shape, estimating changes based on a single parameter or empirical description of the morphological shape changes between healthy and diseased hearts. As such, no previous study has provided an objective, detailed characterization of $R V$ shape changes in health vs patients with TR.

In this study, we evaluated the effect of TR on RV shape, and identified whether the major variants in RV shape could be used to identify RVs affected by TR. The study used cardiac magnetic resonance imaging (MRI) to construct three-dimensional RV models and assess shape changes using an open-source shape modeling tool, ShapeWorks [9], which has recently been shown to produce statistically consistent anatomical measurements and clinically relevant population-level shape modes of variations $[10,11]$. We found statistical group differences between the RVs of patients with TR and controls with unprecedented detail. We also identified primary modes of shape variation and determined if these modes could correctly identify patients with TR based on shape differences alone. 


\section{Methods}

\subsection{Patient Cohort Selection}

Patients with TR were identified using the University of Utah medical records database and a combination of procedure and diagnosis codes. Patient imaging data was analyzed from short axis CINE-MRI images, which were acquired as part of routine clinical care [12,13]. All participants and use of clinical data were approved by the University of Utah Internal Review Board. Control MRIs were acquired from a previously collected healthy volunteer cohort at NYU-Langone Medical Center.

\subsection{Model Generation and Shape Analysis}

Short-axis CINE-MRI images were screened for quality and the RV endocardium was manually contoured at end-systole and end-diastole using the Seg3D open-source software package [14]. Contours were created using an implicit model tool to approximate the endocardial border of the right ventricle. The contours were exported as binary segmentations to ShapeWorks [9], where a particlebased shape modeling approach was applied. Specifically, segmentations were pre-processed [10] to place a dense set of homologous landmarks with geometric consistency on each RV endocardial surface. In particular, homologous landmarks (correspondence points) were automatically positioned on RV surfaces by optimizing the information content of the shape model via an entropy optimization scheme that balances the inherent trade-off between the statistical simplicity of the model (i.e., compactness or lowest entropy) and the accuracy of the geometric description of each shape (i.e., good surface samplings) [9]. We then computed statistical group differences [15] to characterize shape changes between the control and TR groups.

\subsection{Statistical Shape Difference Analysis}

Shape differences were compared between the control and patient groups by computing the mean location of each correspondence point for patient and control groups. These changes were verified using a bootstrap method to account for the varied differences in sample size between groups. Ten bootstrapped models with equal cohorts of six patients and six controls were created for this analysis.

\subsection{Shape-based Prediction Model}

We also constructed a shape-based prediction model to identify control vs. patient RV shapes. To build this model, we performed principal component analysis (PCA) on the correspondence points and mapped each RV shape to its respective PCA loading vector, which in turn encodes the dominant shape parameters that objectively characterize RV shapes. Our objective was to determine which shape parameters (i.e., PCA loadings) were most predictive of patient vs. control RV shapes. We determined the dimension $K$ of PCA loading vectors using the number of PCA components that explains $99 \%$ of the data variation. Here, we assume $\mathbf{s}_{\mathbf{i}}$ is the PCA loading vector of $K$-entries for shape $i$ and $y_{i}$ is the corresponding clinical label, where $y_{i}$ equal to 1 for patients and 0 for control subjects. We then solved a lasso regression [16] for $N=1000$ random subsets of data, and estimated a weight vector, $\mathbf{w}$

$$
y_{i}=\mathbf{w}^{\mathbf{T}} \mathbf{s}_{\mathbf{i}}+\lambda\|\mathbf{w}\|_{\mathbf{1}},
$$

where $\lambda$ is a regularization parameter. A non-zero entry in w shows the relevance of its respective PCA component to predicting patients. Then, we computed the dominance probability of each PCA component, defined as the number of times it appeared as a non-zero entry in $\mathbf{w}$ divided by $N$. Subsequently, we selected the top three components with the highest probabilities to learn a logistic regression classifier for predicting patients, and to determine the accuracy of classification using these top three components.

\section{Results}

\subsection{Patient Cohort}

Between 2011 and 2019, 1,135 patients with a diagnosis of TR underwent cardiac MRI at the University of Utah. Of these, 41 had moderate or greater TR; 32 had acceptable image quality for analysis. Patient characteristics, including TR etiology and invasive hemodynamics, when available, are listed in Table 1. The TR patient group was on average 59 years old, had left ventricular ejection fraction (LVEF) of $47 \pm 20 \%$, and RVEF of $42 \pm 15 \%$. A right heart catheterization procedure had been performed in 13 of 32 TR patients. The average pulmonary artery (PA) systolic pressure was $45 \pm 18 \mathrm{mmHg}$, and PA diastolic pressure was $21 \pm 7 \mathrm{mmHg}$. In these patients, TR was caused by congenital heart disease (4), pulmonary hypertension (3), left-sided heart failure (8), atrial or ventricular arrhythmia (4), or dilated/restrictive cardiomyopathy (12).

\subsection{Shape Differences}

The mean shape difference between patient and control groups is shown in Figure 1. The average RV shape in patients with TR was more spherical and less triangular, with relative narrowing of the RV base, protrusion of the short axis of the mid-RV, and blunting of the RV apex. The maximum change between groups was an increase of $4 \mathrm{~mm}$ and a decrease of $6 \mathrm{~mm}$ in the overall RV shape. Bootstrapping experiments confirmed these results in magnitude, direction, and location of significant shape changes. 
Table 1. Patient cohort baseline characteristics of interest

\begin{tabular}{cc} 
Characteristic & Mean (std) \\
\hline \hline Male/Female & $9 / 33$ \\
Age & $59(17.7)$ \\
LV Ejection Fraction (\%) & $47(20.2)$ \\
RV Ejection Fraction (\%) & $42(14.6)$ \\
Right Heart Cath & $13 / 33$ \\
PA Systolic (mmHg) & $44.5(20.9)$ \\
PA Diastolic (mmHg) & $18.2(7.1)$ \\
Wedge Pressure (mmHg) & $19.2(6.9)$
\end{tabular}

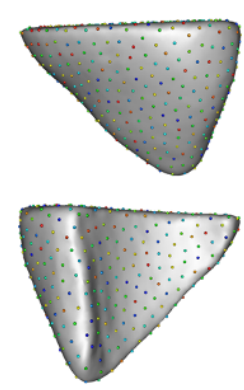

Control Group
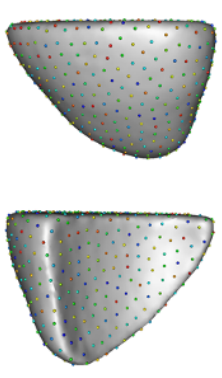

Patient Group

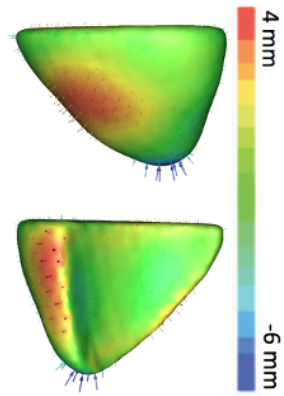

Group Difference
Figure 1. RV shape changes in TR patients vs healthy controls.

\subsection{Shape-based Prediction Model}

We identified 18 modes of variation explaining $99 \%$ of the shape variability between patient and control groups (Figure 2). The three dominant modes of shape variation (modes 3, 5, and 9 in Figure 2) were selected to be used in the prediction model. Mode 3 showed blunting of the RV apex and protrusion of the RV free wall. Mode 5 highlighted relative narrowing of the RV base with widening of the short axis of the mid RV. Mode 9 primarily described widening of the mid RV. Using these three PCA modes, we correctly identified patient vs. control in $86.5 \%$ of cases.

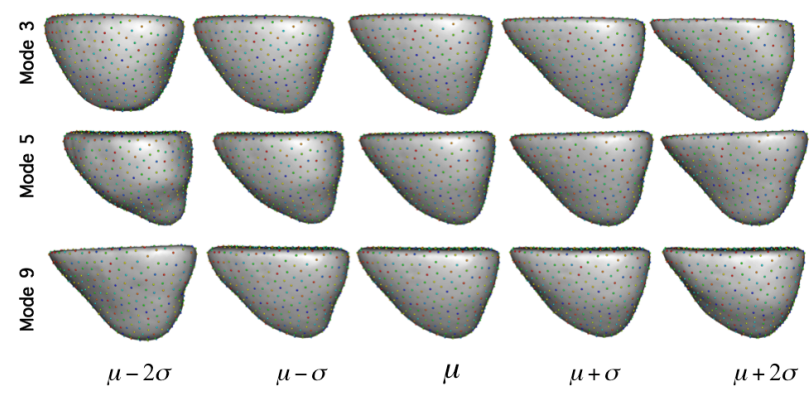

Figure 2. Dominance probability of shape variation modes

\section{Discussion}

In this study, we objectively characterized specific details of RV shape distortion that occur with moderate to severe TR, and confirmed that the primary modes of shape variation can correctly identify these patients by shape alone. Specifically, we first determined the major differences in RV shape between healthy controls and patients with moderate to severe TR. We identified a significant increase in apical curvature $(4 \mathrm{~mm})$ and a significant decrease in inferior apical location $(6 \mathrm{~mm})$. Other studies have previously postulated an increase in RV shape 'sphericity' based on a minimal number of statistical and shape analyses. Our study isolated the specific regions of the RV that are distorted in patients with TR. Our results show that there are specific regions of significant change to RV shape that are indicative of TR.

We also showed that shape analysis can be used as a predictive tool. We found that, by isolating the three modes of highest variation from a PCA analysis, we could correctly identify patients vs. controls $86 \%$ of the time. This finding indicates that shape parameters associated with TR are well defined across a patient population and are identifiable from imaging data. Success in this predictive model suggests a possible shape-based TR diagnosis scheme, which could also be used as a risk stratification tool. Future development of this scheme will focus on the ability to identify shape changes associated with 'minimal' TR compared to severe TR that warrants clinical intervention.

This study has several limitations. First, TR is a dynamic process, which can depend on the volume status of the patient; because this study is retrospective and MRI was obtained at only one time point, it is impossible to know whether the patients had reversible TR and RV dysfunction which may have improved after resolution of a volume overloaded state, vs. fixed dysfunction due to end stage valvular and ventricular changes. Second, only $40 \%$ of the patients included in this study had invasive hemodynamic measurements, with available information regarding pulmonary and RV pressures. It is likely that this group of patients who underwent right heart catheterization were 'sicker', more likely to have significant pulmonary hypertension and RV dysfunction.

These methods have significant potential for future applications in the spectrum of RV disorders and RV failure. Despite the diversity of causes of TR represented in our patient population, RV shape changes followed several predictable patterns, as described above. These patterns were highly selective for identifying images of RVs with TR, relative to controls. Future studies will use the same methods to compare patients with similar etiologies of RV dysfunction but without TR, to the current patient population; we anticipate that such patients may have subtle RV shape changes that represent an earlier point on the spec- 
trum of RV dysfunction, relative to the more advanced disease shown in the TR cohort. Furthermore, these images are ideally suited to future mechanics studies, such as the creation of finite element models, which will correlate the nuances of RV shape change with specific mechanical dysfunction to create a complete picture of RV disease.

\section{Acknowledgments}

This work was supported by the National Institutes of Health under grant numbers NIBIB-U24EB029011, NIAMS-R01AR076120, NHLBI-R01HL135568 (Elhabian), and NIGMS-P41GM103545 (MacLeod), NIH F30HL149327 (Zenger), NIH R01-HL128278 (Weinsaft), NIH 5K-23-HL140092-03 (Kim). The content is solely the responsibility of the authors and does not necessarily represent the official views of the National Institutes of Health.

\section{References}

[1] Singh JP, Evans JC, Levy D, Larson MG, Freed LA, Fuller DL, Lehman B, Benjamin EJ. Prevalence and clinical determinants of mitral, tricuspid, and aortic regurgitation (The Framingham Heart Study). American Journal of Cardiology 1999;ISSN 00029149.

[2] Topilsky Y, Maltais S, Medina Inojosa J, Oguz D, Michelena H, Maalouf J, Mahoney DW, Enriquez-Sarano M. Burden of Tricuspid Regurgitation in Patients Diagnosed in the Community Setting. JACC Cardiovascular Imaging 2019; ISSN 18767591.

[3] Fender EA, Petrescu I, Ionescu F, Zack CJ, Pislaru SV, Nkomo VT, Cochuyt JJ, Hodge DO, Nishimura RA. Prognostic Importance and Predictors of Survival in Isolated Tricuspid Regurgitation: A Growing Problem. Mayo Clinic Proceedings 2019; ISSN 19425546.

[4] Nath J, Foster E, Heidenreich PA. Impact of Tricuspid Regurgitation on Long-Term Survival. Journal of the American College of Cardiology 2004;ISSN 07351097.

[5] Fender EA, Zack CJ, Nishimura RA. Isolated tricuspid regurgitation: Outcomes and therapeutic interventions. Heart 2018;ISSN 1468201X.

[6] Leary PJ, Kurtz CE, Hough CL, Waiss MP, Ralph DD, Sheehan FH. Three-dimensional analysis of right ventricular shape and function in pulmonary hypertension. Pulmonary Circulation 2012;ISSN 20458940.

[7] Mauger C, Gilbert K, Lee AM, Sanghvi MM, Aung N, Fung K, Carapella V, Piechnik SK, Neubauer S, Petersen SE, Suinesiaputra A, Young AA. Right ventricular shape and function: Cardiovascular magnetic resonance reference morphology and biventricular risk factor morphometrics in UK Biobank. Journal of Cardiovascular Magnetic Resonance 2019;ISSN 1532429X.

[8] Farrar G, Suinesiaputra A, Gilbert K, Perry JC, Hegde S, Marsden A, Young AA, Omens JH, McCulloch AD. Atlasbased ventricular shape analysis for understanding congenital heart disease, 2016.
[9] Cates J, Elhabian S, Whitaker R. ShapeWorks: ParticleBased Shape Correspondence and Visualization Software. Statistical Shape and Deformation Analysis January 2017; 257-298.

[10] Goparaju A, Bone A, Hu N, Henninger HB, Anderson AE, Durrleman S, Jacxsens M, Morris A, Csecs I, Marrouche $\mathrm{N}$, et al. Benchmarking off-the-shelf statistical shape modeling tools in clinical applications. arXiv preprint arXiv200902878 2020;

[11] Goparaju A, Csecs I, Morris A, Kholmovski E, Marrouche N, Whitaker R, Elhabian S. On the evaluation and validation of off-the-shelf statistical shape modeling tools: a clinical application. In International Workshop on Shape in Medical Imaging. Springer, 2018; 14-27.

[12] Abouzeid CM, Shah T, Johri A, Weinsaft JW, Kim J. Multimodality Imaging of the Right Ventricle, 2017.

[13] Marcu CB, Beek AM, Van Rossum AC. Cardiovascular Magnetic Resonance Imaging for the Assessment of Right Heart Involvement in Cardiac and Pulmonary Disease. Heart Lung and Circulation 2006; ISSN 14439506.

[14] CIBC, 2016. Seg3D: Volumetric Image Segmentation and Visualization. Scientific Computing and Imaging (SCI) Institute, SLC, UT, USA, Download from: http://www.seg3d.org.

[15] Cates J, Fletcher PT, Whitaker R. A hypothesis testing framework for high-dimensional shape models. MFCA'08 $; 170$.

[16] Tibshirani R. Regression shrinkage and selection via the lasso. Journal of the Royal Statistical Society Series B Methodological 1996;58(1):267-288.

Address for correspondence:

Ashley Morgan, MD

Division of Cardiothoracic Surgery

University of Utah

30 North 1900 E, SOM 36-127

Salt Lake City, Utah 84132

Ashley.Morgan@hsc.utah.edu 